\title{
Reflexión sobre la motivación de los alumnos de Grado en la elección de estudios de comunicación en las universidades de Madrid
}

Fernando Peinado Miguel

Universidad Complutense de Madrid peinado@ccinf.ucm.es

Manuel FERNÁNDEZ SANDE

Universidad Complutense de Madrid

manuel.fernandez@ccinf.ucm.es

Recibido: $14 / 03 / 2011$

Aceptado: 16/09/2011

\section{Resumen}

Este trabajo es una reflexión consecuencia de la investigación que un grupo de profesores del Departamento de Periodismo IV (Empresa Informativa) de la UCM acometen en julio de 2010 a partir de una muestra consolidada de trescientos alumnos que participan en la Summer Media School y cuyo objetivo primordial era su alfabetización multimedia y su familiarización con los procesos productivos en los mass media. Entre los objetivos está el conocer qué motivos les impulsaba a estos alumnos a cursar algunos de los Grados en Comunicación en las Universidades de Madrid, a partir de veinte ítems que surgen de una de las treinta y cuatro preguntas del cuestionario: ¿Porque has elegido estudiar esta titulación superior?

Palabras clave: Comunicación, universidad, motivación, consumo de Medios, EEES

Reflection on the motivation of undergraduate students in the choice of communication studies at the universities of Madrid

\begin{abstract}
This paper reflects the research result of a group of professors of Department of Journalism IV (Media Management) of the UCM in July 2010 from a sample consolidated the three hundred students participating in the Summer Media School and whose main objective was their media literacy and familiarity with production processes in the mass media. Among the objectives is to know what reasons drove them to these students attend some of the degrees in Communication at the University of Madrid, from twenty items arising from one of the thirty-four survey questions: Why have you chosen to study this higher degree?
\end{abstract}

Keywords: Communication, University, Motivation, Media Consumption, EHEA.

\section{Referencia normalizada}

PEINADO MIGUEL, Fernando y FERNÁNDEZ SANDE, Manuel (2011): "Reflexión sobre la motivación de los alumnos de Grado en la elección de estudios de comunicación en las universidades de Madrid”. Estudios sobre el mensaje periodístico. Vol. 17, núm. 2, págs.: 383-400. Madrid, Servicio de Publicaciones de la Universidad Complutense.

Sumario: 1. Preocupaciones que motivan la investigación. 2. Metodología. 2.1. La selección de la muestra. 2.2. Procedimiento e instrumentos utilizados. 3. Resultados del Estudio. 3.1. Factores de motivación extrínseca. 3.2. Factores de motivación intrínseca. 3.3. Motivos de logro y prestigio. 3.4. Motivos relacionados con la filiación y el interés con las relaciones. 4. Conclusiones. 5. Referencias bibliográficas. 6. Anexos: tablas estadísticas de valoración según el estudio.

\section{Preocupaciones que motivan la investigación}

Es este un momento de crisis, y de cambio. Una época para pensar, y preocuparse. Pero también es tiempo de buscar soluciones, de darnos ánimos e intentar motivar a cuantos, desde su debilidad, pueden poner en duda su futuro. 
Este trabajo es una reflexión que nos permite acercarnos a esos alumnos que han tomado una decisión en un periodo de su vida en el que no están todavía lo suficientemente maduros como para acertar con la elección. Alumnos que se han matriculado en algunas de las licenciaturas, grados y dobles titulaciones en Ciencias de la Información y de la Comunicación que se ofertan desde las Universidades, públicas y privadas, que hay en la comunidad de Madrid. Estudiantes que quieren una formación superior para poder ejercer una profesión cualificada.

¿Qué razones tienen para elegir estudiar en uno u otro centro? ¿Por qué van a optar por esta titulación universitaria? ¿Qué esperan de estos estudios superiores y qué objetivos desean satisfacer? Son algunas de las preguntas que nos hicimos los profesores que íbamos a impartir docencia por primera vez en $1^{\circ}$ de Grado de Periodismo, acostumbrados a tratar con alumnos de $2^{\circ}$ ciclo de licenciatura y Posgrado. A principios de 2010 nos ocupamos de preparar ese cambio y diseñamos una estrategia docente e investigadora que se repartía en:

a) realizar un estudio científico sobre el terreno una vez iniciado el curso 20102011, a partir del Proyecto número 247 de Innovación y Mejora de la Calidad Docente de la Universidad Complutense de Madrid ${ }^{1}$ (Peinado et al, 2011) y

b) en julio de 2010, ese mismo grupo de profesores ${ }^{2}$ del Departamento de Periodismo IV, Empresa Informativa, de la Facultad de Ciencias de la Información de Madrid de la Universidad Complutense de Madrid, iniciamos una investigación entre alumnos que deseaban cursar alguno de los Grados en Comunicación en las Universidades de Madrid.

Desde el principio, la idea consistía en conocer cuál era el perfil de esos próximos alumnos de Periodismo, de Comunicación Audiovisual y de Publicidad y Relaciones Públicas, y las motivaciones que les impulsaban para llevar a cabo esa travesía universitaria superior, además de buscar su relación con los medios de comunicación y la influencia que estos ejercen a la hora de crear expectativas profesionales, sin tener en cuenta las redes sociales como principal órgano conversador y de información de ciertos grupos de jóvenes (TAPIA et al, 2010: 3-4)

No nos vamos a engañar. Estamos ante una situación francamente desagradable del mercado laboral de la comunicación no sólo en España (WEAVER y WILLNAT, 2011: web) motivada por la crisis financiera y el arrastre económico del sector. Los principales problemas para quienes desean ejercer profesionalmente cualquiera de las actividades relacionadas con el Periodismo, la Comunicación Audiovisual y la Publicidad y las Relaciones Públicas son: el intrusismo laboral, la baja remuneración, el paro, la inestabilidad laboral, la incompatibilidad con la vida familiar, todos ellos paradigma del pasado 2010 (FARIAS BATLLE, 2010a: 15) Otras preocupaciones como la falta de

${ }^{1}$ Peinado Miguel, F. (IP); Fernández Sande, M.; Ortiz Sobrino, MA; Rodríguez Barba, D., Profesores de "Teoría de la Empresa Informativa", $1^{\circ}$ de Grado de Periodismo. Periodismo IV, UCM.

${ }^{2}$ Ortiz Sobrino, MA (como IP); Rodríguez Barba, D.; Fernández Sande, M. y Peinado MiGUEL, F. son los partícipes del proyecto OTRI de la UFV para la Fundación FIDES (2010) Perfil de entrada de los alumnos de Comunicación en la Universidad de Madrid. 
independencia, la falta de prestigio o la baja cualificación que en 2009 estaban entre los diez problemas más importantes han desaparecido por motivos obvios. Una realidad que los jóvenes aspirantes desconocen cuando toman su elección.

Vivimos en una sociedad en las que la percepción de la realidad es diferente según quienes sean los encuestados. Como muestra un botón, en lo que respecta a la profesión periodística. Tenemos datos poblacionales que nos señalan a la Televisión como el medio de mayor credibilidad (FARIAS BATLLE, 2010b: 106); Las encuestas a los periodistas realizadas en el mismo estudio ${ }^{3}$ consideran a la Radio como el medio más creíble. A su vez, los resultados del estudio realizado a los futuros alumnos de Periodismo reflejan que el medio con mayor credibilidad es la prensa. Ante tanta disparidad en los resultados, entendemos una muestra más de preocupación a la hora de afrontar cuáles son los intereses y deseos de estos alumnos, futuros profesionales.

En el caso del Periodismo, posible espejo de todo cuanto acontece en el sector de la comunicación, cuya práctica parece estar en crisis y con un mercado laboral en franco retroceso, "las cifras de la evolución de empleo que marcan pautas referenciales hacia donde se encamina el futuro de la figura del periodista. Las tendencias globales de empleo marcan curvas bien diferentes en la oferta global y en la oferta para periodistas" (TúÑEZ et al, 2010: 81) A lo mejor tenemos que replantearnos, como principal estrategia, los estudios de esta titulación en la actualidad (CALCUTT y HAMMOND, 2011) en cuanto al atractivo que pueden generar y los nuevos retos tecnológicos a los que se va a enfrentar. Pero no se debe dejar en el saco del olvido que la formación es fundamental y que el futuro del Periodismo pasa por la educación superior de los interesados (FrANKLIN, 2011) Principalmente desde la entrada en vigor del Espacio Europeo de Educación Superior (EEES) en el que "la acumulación de conocimiento pierde relevancia en la sociedad actual en favor de una cualificación más integral, entendida no sólo como un equilibro entre el acopio de información o conocimiento y el dominio de destrezas profesionales" (TúÑEZ et al, 2010: 87)

Somos conscientes que en las facultades no se hacen periodistas, ni publicitarios, ni directores de cine. En las Facultades de Comunicación hacemos licenciados (PEINADO, 2011). Licenciados en Periodismo, en Comunicación Audiovisual, en Publicidad y Relaciones Públicas a los que debemos enseñar a pensar, de forma científica, para encontrar soluciones y aportar a la sociedad desde su posición formativa cualificada. Lo mismo ocurre en la Facultad de Derecho, en la que, por supuesto, no se hacen abogados. Para ser periodista, para ejercer la profesión periodística, como en la mayoría de las profesiones, es fundamental la vocación. Pero, además, hay que ejercer el oficio. Hay que conocerlo, practicarlo, aprenderlo. Y "la profesión, no se nos puede olvidar, es ante todo una actividad humana que se desenvuelve en el ámbito de la sociedad" (REAL, 2009: 98)

${ }^{3}$ El Informe Anual de la Profesión Periodística de la APM es uno de los resultados del Proyecto I+D+i CSO2008-05125 del Ministerio de Ciencia e Innovación, del que FARIAS BATLLE es el IP y en el que participan como investigadores los autores de este artículo. 


\section{Metodología}

Una decisión tan trascendente como la elección de una carrera universitaria responde a diferentes estímulos motivacionales de muy diferente naturaleza que condicionan con diversa intensidad en la elección del alumno. Conocer los intereses que mueven a los alumnos a elegir una determinada carrera de comunicación es muy útil para tratar de ofrecer una oferta educativa que satisfaga lo máximo posible esas expectativas. El objetivo de nuestro estudio era determinar los factores de motivación que podríamos considerar de primer orden para los futuros estudiantes de comunicación. Seleccionamos un conjunto de 20 ítems que se corresponden con una meta u objetivo personal. Seguimos la concepción de meta como una "representación cognitiva de qué es lo que un individuo está intentando conseguir en una situación dada" (GÁmEZ y MARRERO, 2003: 122).

\subsection{La selección de la muestra}

La muestra consta de trescientos alumnos, que provenían de diferentes comunidades autónomas y participaban en un curso de verano organizado por la Summer Media School cuyo objetivo primordial era su alfabetización multimedia, el grado de conocimiento del nuevo espacio europeo de educación superior y su familiarización con los procesos productivos en los mass media. Estos futuros estudiantes de Comunicación en la Comunidad de Madrid fueron convocados mediante redes sociales (Twenti y Facebook) y las webs de las entidades organizadoras y colaboradoras: la Federación de Asociaciones de la Prensa (FAPE); la Asociación de la Prensa de Madrid (APM); la Federación de Asociaciones de Radio y Televisión; la agencia de información Servimedia; Microsoft y los ayuntamientos de Pozuelo de Alarcón y de Majadahonda.

La Summer Media School suponía la primera experiencia en que se tenía ocasión de agrupar a futuros estudiantes de la carrera de comunicación de las diferentes Universidades públicas y privadas de la Comunidad de Madrid. En el curso 2009 / 2010, el número de matriculados tanto en centros públicos como privados en los que se impartían Grados o Licenciaturas de Comunicación, en primera instancia, en Madrid fue de 4310 alumnos. Estos trescientos alumnos eran aspirantes a ocupar una de esas cuatro mil trescientas plazas que se ofertaban en las diferentes Facultades de Comunicación de la Comunidad de Madrid, y por tanto representaban cerca del 10\% del total de estudiantes que han iniciado sus estudios de comunicación en Madrid. Una muestra específica y representativa para poder extraer conclusiones sobre las motivaciones que les han llevado a decidir elegir sus estudios.

Una amplia mayoría de alumnos procedían de la propia Comunidad de Madrid y, a muy larga distancia, también participaron alumnos procedentes de otras Comunidades: Comunidad Autónoma de Valencia, Comunidad Autónoma de Andalucía etc. En cuanto a la composición por sexos la muestra respondía a la proporción habitual en estas carreras con una mayoría de presencia femenina. En el caso de los futuros estudiantes de Periodismo el $67 \%$ eran mujeres y 33\% hombres; para Comunicación Audiovisual 76\% mujeres y $24 \%$ hombres; y Publicidad y Relaciones Públicas $80 \%$ mujeres y $20 \%$ hombres. En cuanto a edades la mayoría de los estudiantes tenían entre 17 y 18 años: el 72\% de los futuros estudiantes de Periodismo, el 58\% de los estudiantes de Comunicación Audiovisual y el $77 \%$ de los de Publicidad y Relaciones Públicas. 
La mayoría de los futuros estudiantes de carreras de comunicación procedían de los estudios de bachillerato, un 67\% en el caso de los futuros estudiantes de Periodismo, un $70 \%$ en los de Comunicación Audiovisual, y un 58\% para los estudiantes de Publicidad.

\subsection{Procedimiento e instrumentos utilizados}

La metodología cuantitativa de la investigación se ha basado en la utilización de un cuestionario de treinta y cuatro preguntas con multirespuesta, con un formato habitual en este tipo de investigaciones (MARTín GonZÁLEZ, 2010: 34-39). A cada uno de los alumnos se les solicitó la colaboración en el estudio y respondió el cuestionario introduciendo sus respuestas en un ordenador con una plantilla de preguntas y respuestas para su posterior tratamiento informático. El trabajo de campo fue realizado entre los días 1 y 15 de julio de 2010, entre las 10 y las 14 horas. Durante la respuesta al cuestionario, estaban asistidos por un operador informático y un responsable del trabajo de investigación que resolvían las dudas de los alumnos.

El cuestionario de 34 preguntas estaba estructurado en diferentes partes: motivación y referencias; consumo y relación; modelos profesionales; influencia de los medios; imagen de la Universidad y Expectativas. En este trabajo presentamos los resultados de la investigación sobre motivación y referencias.

De los 300 cuestionarios cumplimentados por los alumnos, 262 fueron válidos y $38(12,6 \%)$ no pudieron ser analizados por problemas en la grabación de los datos en la aplicación informática, duplicidades y respuestas nulas.

De los 262 cuestionarios procesados: 74 correspondían a futuros estudiantes de Periodismo, 125 a Comunicación Audiovisual y 63 a Publicidad y Relaciones Públicas.

La primera parte del cuestionario dedicada a la motivación de los estudiantes estaba compuesto por una única pregunta ¿Por qué has elegido estudiar Periodismo / Comunicación Audiovisual / Publicidad? El alumno debía contestar a 20 alternativas de respuesta tipo Likert con una gradación desde 1 a 5 :

$1=$ Nada. 2 = Muy poco. $3=$ Algo. $4=$ Bastante. $5=$ Mucho.

Los 20 ítems se referían a la consecución de una meta personal y se incluían metas asociadas a diferentes orientaciones motivacionales. Se incluyeron un grupo de ítems vinculados a los diferentes tipos de metas, con la intención de integrar la dicotomía entre motivación intrínseca y extrínseca, motivación personal y social, motivación de logros y de aprendizaje, etc. que influye en el estudiante cuando toma su decisión. El objetivo de la investigación era identificar los factores que tenían más influencia a la hora de elegir las carreras de comunicación. Para ello identificamos cuatro factores motivacionales que según los autores tienen relación directa con este tipo de decisión (GÁMEZ y MARRERO, 2000: web)

El primer factor, metas asociadas a la motivación externa, compuesto por una serie de cuatro ítems: está de moda estudiar esta carrera; es una carrera fácil; he conocido a algunas personas que han estudiado esta carrera; y mis padres y amigos me lo han aconsejado. La motivación extrínseca está integrada por aquellos estímulos externos a la persona que pueden determinar su conducta. 
El segundo factor incluye objetivos y metas que están ligados a la motivación interna, que se produce cuando las personas actúan para satisfacer necesidades de causación personal y curiosidad. La recompensa se obtiene en la propia realización de la conducta. En nuestro estudio identificamos como motivación intrínseca las metas directamente relacionadas con el aprendizaje -interés por obtener nuevos conocimientos- y el ejercicio de las tareas específicas de la profesión. Este tipo de motivación integraba en nuestro cuestionario cuatro ítems: Me interesa la información de actualidad, contar historias con la imagen y el sonido, organizar eventos de carácter promocional; porque siempre me ha gustado escribir, siempre me ha gustado escribir guiones o hacer programas de radio y TV, siempre me ha gustado la publicidad; porque me atrae el plan de estudios; porque me gustaría aprender a escribir noticias, reportajes o entrevistas, me gustaría aprender la técnica necesaria para trabajar en medios audiovisuales, porque me gustaría aprender a hacer un plan de medios.

El tercer grupo de orientaciones motivacionales pretendía recoger estímulos que están relacionados con la obtención de logros y prestigio, se trata de metas sociales que son tenidas en cuenta en las decisiones individuales. Los estudiantes toman su decisión de elección de unos estudios teniendo en cuenta la posibilidad del éxito profesional y social, fama, prestigio, etc. Se incluyeron seis ítems asociados a este factor: es una profesión que me permitirá ser popular; es la profesión que me permitirá ganar el dinero suficiente para vivir como quiero; porque querría tener un puesto de trabajo con cierto prestigio social; porque hay muchas salidas profesionales para esta carrera; porque me gustaría relacionarme y conocer a personas relevantes; porque admiro a los profesionales que ejercen esta profesión.

Por último nos interesaba también analizar la influencia que tienen en los estudiantes las metas relacionadas con la afiliación e interés por las relaciones sociales. Este grupo comprende los intereses referidos a mejorar sus relaciones, la percepción de sus propias habilidades, la función social de su actividad. Se introdujeron seis ítems: Creo que puedo contribuir a un mundo más justo y mejor; creo que es una profesión nada rutinaria y atractiva; he nacido para ser periodista, profesional de la comunicación audiovisual o de la publicidad no podría dedicarme a otra cosa; porque me gusta trabajar en equipo; porque siempre he sido una persona creativa; porque me gusta conocer otras culturas y países.

A través de los resultados obtenidos de los 20 ítems, queríamos comparar el peso y relevancia otorgada para cada uno de los cuatro factores que integraban las diferentes metas y motivaciones, en la decisión de los estudiantes de elegir cursar estudios superiores de comunicación.

Para profundizar en algunos ítems que formaban parte del cuestionario, se procedió a seleccionar un grupo de 12 futuros estudiantes de carreras de comunicación, respetando siempre la representación proporcional entre los aspirantes a los tres grados, para realizar un focus group cualitativo. En la sesión del grupo de discusión se demandó al alumno una verbalización sobre los aspectos más relevantes, entre ellos se les interrogó sobre sus metas en la elección de los estudios. Las conversaciones resultado del debate fueron transcritas para contrastar algunos datos obtenidos en nuestra investigación cuantitativa. 


\section{Resultados del estudio}

¿Por qué has elegido estudiar esta titulación superior universitaria? Esta era la gran pregunta sobre la que fundamentamos la opción investigadora a partir de elementos cuantitativos y cualitativos y por los que requeríamos explicaciones a partir de modelos motivacionales. La motivación está considerada como una variable importante en el aprendizaje y, desde enfoques académicos, es considerada un "constructor hipotético que explica el inicio, dirección, perseverancia de una conducta hacia una determinada meta académica centrado en el aprendizaje, el rendimiento, el yo, la valoración social o la evitación del trabajo" (FUENTE ARIAS, 2002: 72 - 84) Además, en el campo docente e investigador que nos demanda la Empresa Informativa, área en la que nos situamos, estamos acostumbrados a referirnos en nuestros programas teóricos a capítulos que tienen relación directa entre la motivación organizacional y las teorías motivacionales aplicadas a las organizaciones empresariales (GÉLINIER, 1989)

Durante el curso 2001-2002, en la entonces Escuela de Biblioteconomía y Documentación de la UCM (hoy Facultad), se realizó una investigación similar (TEJADA, 2003:195-211) con 250 alumnos matriculados en el primer curso de la Escuela. Esta es una de las diferencias, pues esta investigación se realizó con alumnos que todavía no se habían matriculado, si bien la comparativa establece como principal contraste que aquella tenía un cierto sesgo ya que no analizaba y cotejaba las motivaciones de sus estudiantes con las de los alumnos de otros títulos de Comunicación.

Otro estudio que si nos permite establecer comparativas, además de seguir pautas científicas semejantes, a pesar de hacerse con estudiantes ya matriculados, es el realizado con alumnos de primer curso de Psicología, Derecho y Biología (GÁmEZ y MARRERO, 2003: 121-131) y en que exploraron el papel de las metas sociales, como agentes motivadores de los estudiantes a la hora de elegir estas titulaciones. "El papel de las orientaciones de meta es una línea investigadora de gran actualidad, con aportaciones relevantes en el campo de la motivación de logro y el aprendizaje autorregulado" (FuENTE ARIAS, 2002: 72-84) y representan una unidad de conocimiento estructurada, personal, sobre los intereses de la tarea del logro que ayuda a definir el éxito y la competencia evaluativa.

Cuando nos referimos a metas de tarea estamos ante formas de aprendizaje o de control, apartado en el que observa la motivación intrínseca. Las metas de rendimiento se centran en la capacidad y las que trabajan el "yo" se refieren al logro y el prestigio.

En el caso que nos trae, la causa de acudir a planteamientos motivacionales radicaba en querer definir, conceptualizar y reconocer las razones psicológicas de un grupo a la hora de tomar una decisión, do forma individual. Este estudio está fundamentado en diferentes tendencias o factores que vamos a desarrollar a partir de los resultados del análisis. Partimos de los enfoques motivacionales intrínseco / extrínseco, de logro y prestigio y los relacionados con la filiación y el interés con las relaciones. Siempre a favor de la constatación de una actualidad que el estudio nos deriva respuesta a respuesta.

\subsection{Factores de motivación extrínseca}

En nuestro estudio se incluyeron una serie de ítems que incluían diferentes tipos de 
metas y objetivos que pueden influir en el estudiante, en su toma de la decisión para elegir una carrera, y que corresponden a la llamada motivación extrínseca.

En primer lugar se les propuso la posibilidad de que eligiesen estas carreras porque "está de moda" estudiarlas. De todos los ítems analizados en la investigación podemos concluir que este factor es el menos influyente, según declaran los propios estudiantes. El 68\% de los entrevistados afirma que no influyó nada en su elección, uno de cada seis que muy poco, un 14\% que algo, apenas un 1\% afirma que bastante y ninguno que mucho. Si analizamos los resultados por carreras, podemos observar que para los futuros periodistas el porcentaje de los que dice que pudo influirles algo -un 23\%- es ligeramente más alto que los candidatos de las otras dos carreras.

"El grado de facilidad" de las carreras percibido por los estudiantes tampoco tiene gran incidencia en la decisión. La mitad de los estudiantes considera que no influye nada para elegir estos estudios, el $29 \%$ que muy poco, uno de cada seis piensa que algo y un $1 \%$ contestaron que bastante o mucho. No se aprecian diferencias significativas en este factor en las motivaciones de los estudiantes de las tres titulaciones.

Otro factor de motivación extrínseca que se ha incluido en el estudio es la "presión o influencia" que ha podido ejercer el entorno próximo del estudiante. A la respuesta "He elegido esta carrera porque mis padres y amigos me lo han aconsejado" obtenemos resultados que denotan una escasa influencia. E1 47\% respondió que no fue tenido en cuenta para nada, uno de cada cuatro afirma que muy poco, porcentaje similar al de estudiantes que sí considera que pudo influir algo en su decisión, apenas un $4 \%$ reconoce que se ha visto bastante influido y ningún alumno ha contestado que mucho. En este ítem tampoco se aprecian diferencias significativas entre los resultados agrupados por carreras.

En la decisión de cursar unos estudios superiores también puede tener incidencia "modelos de otras personas conocidas" que ejercen esas profesiones. Ante la respuesta "porque he conocido a algunas personas que han estudiado esta carrera" detectamos que es la meta relacionada con la motivación externa que parece tener mayor importancia para los estudiantes, aunque la mayoría la sigue considerando como poco relevante en su decisión. E1 37\% considera que no incidió nada en su idea de estudios, uno de cada cinco cree que ha modificado muy poco su decisión, el $27 \%$ declara que pudo influir algo, un $15 \%$ que bastante y un $1 \%$ que mucho. Los estudiantes de periodismo son los que tienen más referentes directos de personas conocidas que ejercen esa profesión. Casi la mitad de ellos afirma que el conocer a personas que han estudiado esta carrera pudo influir algo (30\%), bastante (18\%) o mucho $(1 \%)$. Siete puntos porcentuales más que la suma de las tres respuestas para los estudiantes de Comunicación Audiovisual (algo $27 \%$, bastante $14 \%$ y mucho $1 \%$ ). Los estudiantes de publicidad son los que menos conocidos tienen trabajando en la profesión, un $44 \%$ afirma que esas relaciones no influyeron nada en su decisión, uno de cada cinco cree que ha modificado muy poco su decisión, un $24 \%$ que algo, un $13 \%$ que bastante y un $2 \%$ que mucho.

Para los cuatro ítems propuestos relacionados con el llamado factor de motivación extrínseca comprobamos, según declaran los propios estudiantes, que han tenido una escasa influencia en la elección de las carreras de comunicación. A pesar de tratarse de cuestionarios totalmente anónimos es posible que el estudiante oculte una cierta incidencia que estos elementos de influencia externa hayan podido tener en su decisión. 
De hecho, los resultados son muy similares a los obtenidos en otras investigaciones anteriores (GÁMEZ y MARRERO, 2003: 121-131) Para los futuros estudiantes de las carreras de comunicación, al igual que se observó para estudiantes de Derecho, Biología o Psicología, las influencias definidas como motivación extrínseca tienen un peso muy reducido entre las metas y objetivos que les condicionan la elección de sus estudios universitarios (Véase 6. Anexo: Tabla 1. Factor 1. Motivación extrínseca)

\subsection{Factores de motivación intrínseca}

Hemos agrupado como motivación interna una serie de metas y objetivos directamente relacionados con el aprendizaje, el afán de conocimientos y las expectativas referidas a las tareas propias de los estudios y la profesión a la que conducen. En estos ítems el estudiante no atiende a influencias de terceros, no busca ningún tipo de recompensa externa, ni persigue otros objetivos que no se refieran al ánimo de aprender nuevos conocimientos, habilidades y técnicas que le conducirán a ser un buen profesional de la carrera elegida.

Los datos demuestran que las carreras de comunicación son elegidas por los estudiantes con un gran peso de este factor motivacional. En el estudio se plantearon opciones que comprendían este factor desde cuatro perspectivas complementarias: el interés por formarse en las técnicas específicas para ejercer las profesiones de comunicación, el interés expresado hacia algunos de los temas relacionados con estos estudios, la percepción de las habilidades vinculadas al ejercicio de la comunicación y el interés que suscitan los respectivos planes de estudio.

En el primer ítem se personalizó el enunciado para cada una de las carreras en estudio. A los futuros periodistas se les ofrecía la respuesta: "me interesa la información de actualidad"; a los estudiantes de comunicación audiovisual "me interesa contar historias con la imagen y el sonido"; y a los estudiantes de publicidad, para profundizar en los diferentes intereses vinculados a las diversas facetas del licenciado en relaciones públicas y publicidad les pedimos manifestarse sobre cuatro salidas diferentes de esa profesión: "me interesa organizar eventos de carácter promocional", "me interesa todo lo relacionado con el marketing corporativo", "me interesa el mundo de las relaciones públicas", y "me interesa crear spots, anuncios, etc.".

En todas las respuestas los estudiantes manifestaron una influencia alta de estas motivaciones para elegir sus carreras. Así, los estudiantes de comunicación expresan un alto interés en los conocimientos vinculados con estas profesiones a la hora de tomar su decisión. E1 79\% de los futuros periodistas afirmaron que su interés en la información de actualidad había tenido mucho - $24 \%$ - o bastante - $55 \%$ - que ver para que se decantasen por estudiar esta carrera. Un porcentaje ligeramente superior al de los estudiantes de las otras dos carreras. Uno de cada cinco alumnos de comunicación audiovisual afirma estar muy interesado en los conocimientos y tres de cada diez dice tener bastante interés en contar historias con la imagen y el sonido. Si atendemos a los totales de los estudiantes de las tres carreras obtenemos los siguientes resultados: mucho interés $24 \%$, bastante $43 \%$, algo $26 \%$, muy poco $5 \%$ y nada $1 \%$.

Las profesiones vinculadas a los estudios de publicidad se caracterizan por su diversidad y por ello hemos considerado necesario diferenciar las motivaciones que los estudiantes expresan hacia algunos de los conocimientos relacionados. La mayoría de 
estudiantes de publicidad se decantan claramente por el trabajo creativo. Así, el 44\% declara que su interés por crear publicidad -spots, anuncios, etc.- ha influido mucho en su decisión, un 35\% que bastante, un 13\% algo y menos de un $10 \%$ muy poco. El segundo ámbito de intereses es la organización de eventos de carácter promocional uno de cada cinco de los futuros publicitarios consideran que ha incidido mucho en su decisión, casi la mitad afirma que bastante, un $32 \%$ algo, el $8 \%$ muy poco y apenas un $2 \%$ le otorga ningún interés-. Todo lo relacionado con el marketing es la tercera motivación: la mitad de los estudiantes afirma que le interesa mucho o bastante, un $40 \%$ algo y al $10 \%$ muy poco. Por último, el mundo de las relaciones públicas sería la cuarta prioridad según estos resultados, solo un $2 \%$ afirma que ese interés ha influido mucho en su elección de la carrera, en 30\% dice que bastante, un $54 \%$ algo, un $11 \%$ muy poco y un $3 \%$ nada.

Otro de los aspectos investigados es los hábitos relacionados con habilidades muy presentes en el ejercicio profesional. Los futuros profesionales de la comunicación manifestaron que ha influido mucho su afición por estas profesiones. Los estudiantes que quieren cursar periodismo declaran que han elegido esta carrera porque siempre les ha gustado escribir: un $40 \%$ considera que le ha influido mucho, un $34 \%$ que bastante, uno de cada cuatro algo y apenas un $3 \%$ muy poco. Los resultados reflejan unos porcentajes ligeramente superiores que los registrados para las tres carreras: mucho $28 \%$, bastante $34 \%$, algo $28 \%$, muy poco $6 \%$ y nada $4 \%$. En concreto, los alumnos de Comunicación Audiovisual y Publicidad no le otorgan tanto peso a ese ítem en su decisión pero también manifiestan una influencia importante de esta motivación. El 58\% de los de comunicación audiovisual expresa mucha $-26 \%$ - o bastante $-32 \%$ - importancia a escribir guiones o hacer programas de radio y más de la mitad de los de relaciones públicas y publicidad afirma que siempre les ha gustado la publicidad mucho o bastante.

Los planes de estudio de los grados de estas carreras suscitan un interés notable por parte de los estudiantes. En estos resultados sí podemos observar diferencias en la opinión expresada en función de las diferentes carreras. Los estudiantes de publicidad son los que perciben su plan de estudios como más interesante, el $39 \%$ considera que lo atractivo del plan ha condicionado mucho la elección de la carrera, en $43 \%$ dicen que bastante y un $14 \%$ algo. Los futuros periodistas y profesionales de la comunicación audiovisual declaran en su mayoría que el plan lo han tenido en cuenta algo o bastante.

En general y si atendemos a las medias de las tres carreras la aceptación del plan es bastante positiva: uno de cada cinco dice que el plan influyó mucho en su decisión, el $41 \%$ bastante y uno de cada tres algo. Tan solo un $4 \%$ reconoce que el plan les interesa muy poco o nada.

Los resultados más elevados en el apartado de motivación intrínseca los encontramos en el ítem referido a las técnicas y conocimientos específicos que los alumnos esperan obtener en estos estudios. Los estudiantes de las tres carreras eligen sus estudios muy motivados ante la posibilidad de recibir conocimientos que les faculten en las principales técnicas de las profesiones que aspiran ejercer. El 38\% afirma haber influido mucho en su decisión, un $34 \%$ bastante, un $23 \%$ algo, un $3 \%$ muy poco y para 
un $2 \%$ nada. Por carreras, los futuros periodistas quieren aprender a escribir noticias, reportajes o entrevistas - un 38\% expresa que lo han tenido muy en cuenta, idéntico porcentaje que lo ha tenido bastante presente, y un $21 \%$ algo. Por su parte, a los estudiantes de comunicación audiovisual les gustaría aprender las técnicas necesarias para trabajar en medios audiovisuales -para un $40 \%$ esa motivación es muy importante, para un $32 \%$ bastante y para un $24 \%$ algo importante. (Véase 6 . Anexo: Tabla 2. Factor 2. Motivación intrínseca)

\subsection{Motivos de logro y prestigio}

El presupuesto básico de los modelos normativos sostiene que los alumnos pueden ser clasificados según el tipo de meta académica que asuman. Una meta se entiende como la representación cognitiva de Qué es lo que un individuo intenta conseguir ante una situación dada. Este tercer factor incluye seis ítems que están relacionados con metas de autovaloración y agrupa bastante bien los motivos de logro y poder, mediante el que se experimenta el orgullo y la satisfacción que sigue al éxito. Estamos, pues, en una categoría estrechamente ligada al autoconcepto y la autoestima, y se observa como los estudiantes se plantean metas claras de superación personal y en contextos donde puedan garantizar el prestigio y una cierta posición social.

Uno de los grandes problemas que en nuestros días tenemos, sobre todo por el efecto de la televisión, es el fenómeno del "quiero ser famoso". Sin embargo, la pregunta "es una profesión que me permitirá ser popular y trabajar con personajes populares", (no realizada a los que querían estudiar Publicidad y RRPP) se aprecia que los quieren estudiar Periodismo tienen ligeramente en cuenta esta meta (51\% reconoce que les interesa Algo) En la segunda idea, quiero estudiar Periodismo o Comunicación Audiovisual "porque me gustaría relacionarme y conocer a personas relevantes" el porcentaje mayoritario se sitúa en Bastante y Mucho, con unos totales entre ambas titulaciones del $59 \%$.

Las tres titulaciones en el tercer ítem, cuando se refieren a la cuestión económica retributiva, se sitúan entre el Algo y el Muy poco con porcentajes que rozan el 70\% (destaca el $52 \%$ en algo y la tendencia a Bastante de Publicidad) Si el prestigio lo da la retribución económica, es evidente que el motivo no es este. Se refuerza este principio en la siguiente idea: se les pregunta si van a elegir alguno de esos estudios "porque querrían tener un puesto de trabajo con cierto prestigio social" a lo que la mayoría se decantan entre el Nada y el Algo con valores entre el $43 \%$ de periodismo en Algo y $22 \%$ de Comunicación Audiovisual en el Nada, razonamiento que se sitúa en la línea anterior y que permite observar que no es el prestigio lo que motiva su decisión.

Así pues, una de las repuestas que muestran la decisión hacia el logro está en las opciones laborales y salidas profesionales "porque hay muchas para estos estudios" y los porcentajes mayoritarios se orientan hacia el Algo-Bastante, por encima en los tres casos del 70\%. Otro de los factores motivacionales que incitan a la elección es "porque admiro a los profesionales que ejercen esta profesión", en el caso de Periodismo, Bastante-Mucho un 67\%, y cae en Comunicación Audiovisual y en Publicidad al 60\%. Parece que la presencia en medios de comunicación influye directamente en la posibilidad de reconocimiento de la actividad profesional, lo que facilita esa admiración. 
Las metas de logro permiten apreciar el resultado del camino que van a emprender, del esfuerzo que requieren y de como pueden alcanzar el final, para superar el reto que en la mayoría de las ocasiones se comprende desde la visones y del desconocimiento. (Véase 6. Anexo: Tabla 3. Factor 3. Motivos de logro y prestigio)

\subsection{Motivos relacionados con la afiliación y el interés con las relaciones}

Este cuarto factor expresa una motivación orientada a logros asociados a motivos afiliativos y agrupa los deseos y motivos relacionados genuinamente con el poder, para influir sobre los otros, la capacidad de un líder. Entre las metas afiliativas encontramos el como ayudar a los demás, hacer nuevos amigos o ser una persona solidaria. Son valores que facilitan razones específicas para alcanzar objetivos. "Estos fines pueden ser sociales a partir de la individualidad, la autodeterminación, la superioridad y la adquisición de los recursos sociales" (ALONSO TAPIA, 2005: 11-52) y cuando tratan relaciones integradoras, se refieren al logro d relaciones comunes, responsabilidad y compromisos sociales, la equidad o la justicia y la provisión de recursos sociales, como se refleja en el primero de los ítems de este grupo "creo que como periodista puedo contribuir a un mundo más justo y mejor" tanto los de Periodismo como los de Comunicación Audiovisual responden por encima del 50\% entre Bastante y Mucho. A los de Publicidad se les plantea "creo que como profesional publicitario voy a contribuir a un consumo más justo" y el 64\% es Bastante-Mucho.

La segunda cuestión también decanta su elección en porcentaje muy altos. "Es una profesión nada rutinaria y muy atractiva" obtiene porcentajes que superan el $80 \%$ en Bastante y Mucho ( $41 \%$ en Periodismo) La dinamicidad laboral y el famoso quiero ser reportero de guerra parece que se mantiene vivo. Ya en el cualitativo (Focus Group) se comprueba que lo dinámico de la profesión es lo más atractivo. No desean profesiones rutinarias y por supuesto nada de despachos.

El 34\% dice que Algo de vocación tienen par dedicarse al Periodismo y un 24\% Bastante. El porcentaje de Comunicación Audiovisual es superior (34+24+18 Mucho) e incluso en Publicidad, el 38\% en Bastante supera a las otras opciones. Es evidente que han nacido para dedicarse a estas profesiones, tal y como expresan en el cualitativo "es la única carrera que me motiva", si bien con pequeños reparos.

Todos están acostumbrado o al menos aseguran que les gusta trabajar en equipo, con porcentajes muy altos, en Bastante: $45 \%$ en Comunicación Audiovisual; 39\% Periodismo y $41 \%$ Publicidad. Precisamente todos se sienten muy creativos y consideran que es motivo para elegir estos estudios, desde el $42 \%$ de Periodismo en Bastante al $43 \%$ de Publicidad en Mucho. Por último, para elegir Periodismo, el 57\% es Mucho (gente viajera) y el $42 \%$ en Comunicación Audiovisual, por un 36\% en Publicidad. Se acuerda una relación con la dinamicidad, la falta de rutina y la vocación. (Véase 6 . Anexo: Tabla 4. Factor 4. Motivos relacionados con la afiliación y el interés por las relaciones)

\section{Conclusiones}

Partimos de factores que se encuentran en relación con la motivación para entender cuáles son las razones que han provocado a estos estudiantes la elección de una titulación universitaria en el marco de la Comunicación y qué objetivos esperan satisfacer como consecuencia de esa decisión. Además, los estudiantes que se incorporan a 
la Universidad en el primer año se encuentran con un mundo nuevo, diferente. Es un espacio en el que inician una vida que les hace dejar atrás la adolescencia, razón por la que necesitan integrarse y ser aceptados por el nuevo grupo, además de sentir la preocupación que las nuevas relaciones van a provocarles.

Tras analizar los resultados observamos una serie de respuestas que pueden sernos de enorme utilidad para aconteceres presentes y futuros relacionados con la docencia en cualquiera de los grados de Comunicación.

1. El perfil de los estudiantes de las tres titulaciones de comunicación presenta muchas coincidencias en lo referente a sus motivaciones y objetivos. Podemos encontrar ligeras diferencias en el peso de una u otra motivación, pero en términos generales se sienten influidos por los mismos factores. Esto refuerza la relación entre los gustos e intereses que manifiestan los futuros alumnos de comunicación con independencia de su especialización. La tendencia a una conjunción transversal de materias y conocimientos que se refleja, precisamente, en los nuevos planes de estudio relativos a los Grados de Periodismo, Comunicación Audiovisual y Publicidad y Relaciones Públicas, y que nacen como respuesta al nuevo Espacio Europeo de Educación Superior. Se puede aventurar que la capacidad discente de los estudiantes de cualquiera de estas tres titulaciones les puede ofrecer múltiples y paralelas salidas en un mismo mercado, el de la comunicación.

Y resulta sorprendente el enorme atractivo que los planes de estudio de los grados de comunicación conllevan entre los estudiantes a la hora de elegir su opción. Lamentablemente no tenemos estudios previos para poder comparar esos resultados, mas sería interesante saber si la oferta de los nuevos grados resulta de mayor interés y seducción que los anteriores planes de estudios. Destacar que son los futuros estudiantes de publicidad los que se muestran más ilusionados.

Pero todos consideran que van a recibir una formación basada en técnicas específicas vinculadas a las profesiones, apreciación que se refuerza en el cualitativo: el aprender la profesión. Demanda que no sorprende por ser muy habitual en los estudiantes de estas titulaciones, desconocedores de una realidad docente que en los próximos años debemos cambiar.

2. Podemos señalar que el peso observado de los diferentes factores de motivación no difiere en exceso, especialmente en algunas de las metas analizadas, de otros estudios similares al que aquí se muestra, aunque se puedan haber aplicado otras metodologías. Por lo tanto, no se ha inventado nada, aunque si se confirma lo que hasta ahora se venía estudiando, con alguna alteración adaptativa. El factor de la "motivación externa", según expresan los futuros estudiantes de comunicación, es el que menos les influye a la hora de decidir los grados que quieren realizar. Su decisión no tiene que ver con moda alguna, ni está en relación con el grado de dificultad de los estudios y, no es consecuencia de los consejos de sus padres y amigos. Los jóvenes que van a comenzar estos estudios superiores se mueven con mucha mayor intensidad por otro tipo de motivaciones más personales.

3. El grupo de ítems que tampoco tiene apenas incidencia en la toma de decisiones para elegir una titulación en Comunicación es el de "motivos de logro y prestigio". Los futuros estudiantes de comunicación declaran que el dinero que puedan ganar, las 
salidas profesionales o el prestigio que puedan alcanzar no resultan para ellos lo más determinante y le dan prioridad a otros objetivos. Se aprecian algunas diferencias entre los estudiantes de las tres titulaciones. Por ejemplo, que los futuros estudiantes de Periodismo reconocen un interés ligeramente mayor que los de los otros estudios cuando valoran la importancia que tiene para ellos alcanzar popularidad o relacionarse con personas relevantes. Los estudiantes de Publicidad y Relaciones Públicas son el grupo que declara un interés superior ante la remuneración económica que comporta su esfuerzo laboral. La meta, relacionada con el logro y prestigio a alcanzar, que obtiene unos porcentajes más altos por su influencia es la admiración que sienten los estudiantes por los profesionales que ejercen su actividad en este sector. Para la mayoría de los futuros matriculados en alguno de los tres grados esa admiración ha podido influir de forma considerable.

4. Los futuros estudiantes de comunicación manifiestan un gran interés por recibir formación en temas que consideran centrales en el ejercicio de esas profesiones: la información de actualidad, la narrativa audiovisual o la creación de spots y anuncios. Es este un momento en el que existe esa gran oportunidad de satisfacer ese interés y por otro una importante responsabilidad de no defraudarles en esas expectativas. Otra motivación que ejerce una gran influencia en su decisión es la afición que manifiestan por la escritura o la publicidad desde años atrás. En el focus group los estudiantes también manifestaron en su mayoría que desde pequeños habían tenido interés por estas profesiones y que habían decidido que querían estudiarlas desde antes del bachillerato, lo que confirma esa vocación temprana (y necesaria) en muchos casos. Por último, resaltar el momento de conciencia global, en el que estos futuros estudiantes de comunicación otorgan un gran peso para elegir sus estudios a factores motivacionales que tienen vinculación con su posible contribución a mejorar la sociedad (mediante aportaciones solidarias y con elementos de equidad en el consumo) a la naturaleza dinámica y creativa de los trabajos elegidos, a la posibilidad de conocer otras culturas y países. Y todo ello con un alto interés por trabajar en equipo, habilidad necesaria para integrarse en las estructuras empresariales.

\section{Referencias bibliográficas}

ALONSO TAPIA, Jesús. (2005): "Motivación para el aprendizaje: la perspectiva de los alumnos", en Foro Educacional, n ${ }^{\circ}$ 7. Chile, Ediciones Universidad Católica Silva Henríquez, pp. 11-52.

CALCUTT, Andrew y HAMMOND, Philip. (2011): Journalism Studies. A critical introduction. London, Routledge.

FARIAS BATLLE, Pedro. (2010a): "La credibilidad, el mejor salvavidas", en Informe Anual de la Profesión Periodística 2010. Madrid, APM, pp. 15-19.

FARIAS BATLLE, Pedro. Director (2010b): Informe Anual de la Profesión Periodística 2010. Madrid, APM, pp. 99-118.

FORD, Martin E. (1992): Motivating humans: Goals, emotions and personal agency beliefs. Newbury Park, CA: Sage. 
FRANKLIN, Bob. (2011): The Future of Journalism. Cardiff University, UK.

FUENTE ARIAS, Jesús (2002): "Perspectivas recientes en el estudio de la motivación: la Teoría de la orientación de la meta", en Escritos de Psicología, $\mathrm{n}^{\circ}$ 6. Universidad de Málaga, pp. 72-84.

GÁMEZ, Elena y MARRERO, Hipólito (2000): "Metas y motivos en la elección de la carrera de psicología", en Revista Electrónica de Motivación y Emoción R.E.M.E-, Vol. 3, $n^{\circ}$ 5-6. Universitat Jaume I. Disponible en http://reme.uji.es. (Fecha de consulta: 12 de marzo de 2011)

GÁMEZ, Elena y MARRERO, Hipólito (2003): "Metas y motivos en la elección de la carrera universitaria: Un estudio comparativo entre psicología, derecho y biología", en Anales de Psicología, Vol. 19, n 1 . Servicio de Publicaciones de la Universidad de Murcia, pp. 121-131.

GÉLINIER, Octave. (1989): Estrategia y Motivación. Barcelona, Civilización Ediciones.

MARTÍN GONZÁLEZ, José Antonio (2010) "Cinco consejos para la investigación cuantitativa", en Marketing+ Ventas, $\mathrm{n}^{\circ}$ 258. Editorial Wolters Kluwer España, pp. 34-39, http://marketingmasventas.wke.es/. (Fecha de consulta: 8 de marzo de 2011)

ORTÍZ SOBRINO, Miguel Ángel y RODRÍGUEZ BARBA, María Dolores (2011): "El perfil de entrada de los alumnos de grado en las facultades de comunicación de las universidades de Madrid: el caso de la Summer Media School de la fundación Fides/UFV", en Vivat Academia, $\mathrm{n}^{\circ}$ 114. Madrid, www.ucm.es/info/vivataca. (Fecha de consulta: 15 de marzo de 2011)

PEINADO MIGUEL, Fernando (2011): "Motivación + Innovación + Bolonia = Formación Superior Necesaria", en Anuario de la Comunicación 2011. Madrid, Dircom.

PEINADO MIGUEL, Fernando; FERNÁNDEZ SANDE, Manuel; ORTÍZ SOBRINO, Miguel Ángel y RODRÍGUEZ BARBA, María Dolores (2011): "Hacia un aprendizaje activo de la empresa informativa en el EEES. Aplicación del podcasting y otras herramientas de comunicación 2.0", en Razón y Palabra, $\mathrm{n}^{\circ} 75$. México, http://www.razonypalabra.org.mx [Fecha de consulta: 10 de marzo d 2011]

REAL, Elena (2009): "La identidad del periodista en el futuro Estatuto profesional, entre la confusión y la desprofesionalización", en Estudios sobre el Mensaje Periodístico, $\mathrm{n}^{\mathrm{o}}$ 15. Madrid, Servicio de Publicaciones de la Editorial Complutense, pp. 95-118.

TAPIA, Alejandro; GÓMEZ NIETO, Begoña; HERRANZ, José María y MATELLANES, Mónica (2010): "Los estudiantes universitarios ante las redes sociales: cuestiones de uso y agrupamiento en estructuras elitistas o pluralistas", en Vivat Academia, $\mathrm{n}^{\circ}$ 113. Madrid, www.ucm.es/info/vivataca. (Fecha de consulta: 9 de marzo de 2011)

TEJADA, Carlos (2003): "Perfil, motivaciones y expectativas de los alumnos de primer curso de la escuela de Biblioteconomía y Documentación de la UCM", en Re- 
vista General de Información y Documentación, $\mathrm{n}^{\mathrm{o}}$ 13. Madrid, Servicio de Publicaciones de la Editorial Complutense, pp. 195-211.

TÚÑEZ, Miguel; MARTÍNEZ SOLANA, Yolanda y ABEJÓN MENDOZA, Paloma: (2010): "Nuevos entornos, nuevas demandas, nuevos periodistas", en Estudios sobre el Mensaje Periodístico, $\mathrm{n}^{\circ} 16$. Madrid, Servicio de Publicaciones de la Editorial Complutense, pp. 79-94.

WEAVER, David H. y WILLNAT, Lars (2011): The global Journalist in the 21st Century. Presentación del estudio en la Facultad de Ciencias de la Comunicación de la UMA, el 11 de enero. Editado por la Universidad de Indiana, EEUU, en agosto 2011, en: http://www.uma.es/contenido.php?idm=29\&clase $=$ p\&id $=2245 \&$ tipo $=n$

\section{Anexos: tablas estadísticas de valoración según el estudio}

\begin{tabular}{|c|c|c|c|c|c|}
\hline Tabla 1. Factor 1. Motivación extrínseca & \multirow{2}{*}{ Nada } & \multirow{2}{*}{ Muy Poco } & \multirow{2}{*}{ Algo } & \multirow{2}{*}{ Bastante } & \multirow{2}{*}{ Mucho } \\
\hline Porque está de moda estudiarla & & & & & \\
\hline Periodismo & $61 \%$ & $15 \%$ & $23 \%$ & $1 \%$ & $0 \%$ \\
\hline Comunicación Audiovisual & $72 \%$ & $16 \%$ & $11 \%$ & $0 \%$ & $1 \%$ \\
\hline Publicidad & $68 \%$ & $21 \%$ & $10 \%$ & $2 \%$ & $0 \%$ \\
\hline Total Estudiantes & $68 \%$ & $17 \%$ & $14 \%$ & $1 \%$ & $0 \%$ \\
\hline \multicolumn{6}{|l|}{ Porque es una carrera fácil } \\
\hline Periodismo & $54 \%$ & $30 \%$ & $16 \%$ & $0 \%$ & $0 \%$ \\
\hline Comunicación Audiovisual & $57 \%$ & $23 \%$ & $18 \%$ & $1 \%$ & $2 \%$ \\
\hline Publicidad & $41 \%$ & $40 \%$ & $16 \%$ & $2 \%$ & $2 \%$ \\
\hline Total Estudiantes & $52 \%$ & $29 \%$ & $17 \%$ & $1 \%$ & $1 \%$ \\
\hline \multicolumn{6}{|c|}{ Porque mis padres y amigos me lo han aconsejado } \\
\hline Periodismo & $41 \%$ & $31 \%$ & $24 \%$ & $3 \%$ & $1 \%$ \\
\hline Comunicación Audiovisual & $49 \%$ & $22 \%$ & $23 \%$ & $6 \%$ & $0 \%$ \\
\hline Publicidad & $52 \%$ & $17 \%$ & $29 \%$ & $2 \%$ & $0 \%$ \\
\hline Total Estudiantes & $47 \%$ & $24 \%$ & $25 \%$ & $4 \%$ & $0 \%$ \\
\hline \multicolumn{6}{|c|}{ Porque he conocido algunas personas que estudian esta carrera } \\
\hline Periodismo & $34 \%$ & $18 \%$ & $30 \%$ & $18 \%$ & $1 \%$ \\
\hline Comunicación Audiovisual & $36 \%$ & $22 \%$ & $27 \%$ & $14 \%$ & $1 \%$ \\
\hline Publicidad & $44 \%$ & $17 \%$ & $24 \%$ & $13 \%$ & $2 \%$ \\
\hline Total Estudiantes & $37 \%$ & $20 \%$ & $27 \%$ & $15 \%$ & $1 \%$ \\
\hline \multicolumn{6}{|c|}{ A partir de los resultados del cuantitativo, julio 2010, elaboración propia. } \\
\hline Tabla 2. Factor 2. Motivación intrínseca & & & & & \\
\hline $\begin{array}{l}\text { Me interesa la información de actuali- } \\
\text { dad/contar historias con imagen y so- } \\
\text { nido/organizar eventos }\end{array}$ & Nada & Muy Poco & Algo & Bastante & Mucho \\
\hline Periodismo & $1 \%$ & $0 \%$ & $19 \%$ & $55 \%$ & $24 \%$ \\
\hline Comunicación Audiovisual & $2 \%$ & $7 \%$ & $26 \%$ & $37 \%$ & $28 \%$ \\
\hline Publicidad & $2 \%$ & $8 \%$ & $2 \%$ & $41 \%$ & $17 \%$ \\
\hline Total Estudiantes & $1 \%$ & $5 \%$ & $26 \%$ & $43 \%$ & $24 \%$ \\
\hline
\end{tabular}




\begin{tabular}{|c|c|c|c|c|c|}
\hline \multicolumn{6}{|c|}{ Porque siempre me ha gustado escribir/escribir guiones o hacer programas de radio y tv/la publicidad } \\
\hline Periodismo & $0 \%$ & $3 \%$ & $23 \%$ & $34 \%$ & $40 \%$ \\
\hline Comunicación Audiovisual & $9 \%$ & $8 \%$ & $25 \%$ & $32 \%$ & $26 \%$ \\
\hline Publicidad & $0 \%$ & $6 \%$ & $37 \%$ & $40 \%$ & $17 \%$ \\
\hline Total Estudiantes & $4 \%$ & $6 \%$ & $28 \%$ & $34 \%$ & $28 \%$ \\
\hline \multicolumn{6}{|c|}{ Porque me atrae el plan de estudios } \\
\hline Periodismo & $3 \%$ & $9 \%$ & $37 \%$ & $35 \%$ & $16 \%$ \\
\hline Comunicación Audiovisual & $5 \%$ & $2 \%$ & $34 \%$ & $44 \%$ & $15 \%$ \\
\hline Publicidad & $2 \%$ & $2 \%$ & $14 \%$ & $43 \%$ & $39 \%$ \\
\hline Total Estudiantes & $4 \%$ & $4 \%$ & $30 \%$ & $41 \%$ & $21 \%$ \\
\hline \multicolumn{6}{|c|}{$\begin{array}{l}\text { Porque me gustaría aprender a escribir noticias, reportajes/la técnica necesaria para medios audiovi } \\
\text { suales/ aprender a hacer un plan de mediosa }\end{array}$} \\
\hline Periodismo & $3 \%$ & $0 \%$ & $21 \%$ & $38 \%$ & $38 \%$ \\
\hline Comunicación Audiovisual & $2 \%$ & $2 \%$ & $24 \%$ & $32 \%$ & $40 \%$ \\
\hline Publicidad & $2 \%$ & $10 \%$ & $22 \%$ & $33 \%$ & $33 \%$ \\
\hline Total Estudiantes & $2 \%$ & $3 \%$ & $23 \%$ & $34 \%$ & $38 \%$ \\
\hline
\end{tabular}

\begin{tabular}{|l|c|c|c|c|c|}
\hline Tabla 3. Factor 3. Motivos de logro y prestigio & \multirow{2}{*}{ Nada } & Muy Poco & Algo & Bastante & Mucho \\
\cline { 1 - 4 } $\begin{array}{l}\text { Es una profesión que me permitirá ser po- } \\
\text { pular (y trabajar con personajes populares) }\end{array}$ & & & & & \\
\hline Periodismo & $15 \%$ & $20 \%$ & $51 \%$ & $10 \%$ & $4 \%$ \\
\hline Comunicación Audiovisual & $12 \%$ & $28 \%$ & $42 \%$ & $10 \%$ & $8 \%$ \\
\hline Total Estudiantes & $13 \%$ & $24 \%$ & $47 \%$ & $10 \%$ & $6 \%$ \\
\hline Porque me gustaría relacionarme y conocer a personas relevantes \\
\hline Periodismo & $1 \%$ & $4 \%$ & $24 \%$ & $41 \%$ & $30 \%$ \\
\hline Comunicación Audiovisual & $5 \%$ & $14 \%$ & $35 \%$ & $35 \%$ & $11 \%$ \\
\hline Total Estudiantes & $3 \%$ & $9 \%$ & $29 \%$ & $38 \%$ & $21 \%$ \\
\hline Es la profesión que me permitirá ganar el dinero suficiente para vivir como quiero \\
\hline Periodismo & $11 \%$ & $27 \%$ & $46 \%$ & $13 \%$ & $3 \%$ \\
\hline Comunicación Audiovisual & $8 \%$ & $30 \%$ & $41 \%$ & $20 \%$ & $1 \%$ \\
\hline Publicidad & $16 \%$ & $13 \%$ & $52 \%$ & $17 \%$ & $2 \%$ \\
\hline Total Estudiantes & $12 \%$ & $23 \%$ & $46 \%$ & $17 \%$ & $2 \%$ \\
\hline Porque querría tener un puesto de trabajo con cierto prestigio social \\
\hline Periodismo & $18 \%$ & $27 \%$ & $43 \%$ & $8 \%$ & $4 \%$ \\
\hline Comunicación Audiovisual & $22 \%$ & $29 \%$ & $32 \%$ & $14 \%$ & $3 \%$ \\
\hline Publicidad & $17 \%$ & $24 \%$ & $33 \%$ & $16 \%$ & $10 \%$ \\
\hline Total Estudiantes & $20 \%$ & $27 \%$ & $35 \%$ & $13 \%$ & $5 \%$ \\
\hline Porque hay muchas salidas profesionales para esta carrera & $12 \%$ & $14 \%$ & $34 \%$ & $39 \%$ & $1 \%$ \\
\hline Periodismo & $6 \%$ & $20 \%$ & $43 \%$ & $26 \%$ & $5 \%$ \\
\hline Comunicación Audiovisual & $6 \%$ & $10 \%$ & $48 \%$ & $25 \%$ & $11 \%$ \\
\hline Publicidad & $8 \%$ & $16 \%$ & $41 \%$ & $30 \%$ & $5 \%$ \\
\hline Total Estudiantes
\end{tabular}




\begin{tabular}{|l|c|c|c|c|c|}
\hline Porque admiro a los profesionales que ejercen esta profesión \\
\hline Periodismo & $2 \%$ & $4 \%$ & $27 \%$ & $43 \%$ & $24 \%$ \\
\hline Comunicación Audiovisual & $1 \%$ & $7 \%$ & $32 \%$ & $38 \%$ & $22 \%$ \\
\hline Publicidad & $3 \%$ & $11 \%$ & $36 \%$ & $37 \%$ & $13 \%$ \\
\hline Total Estudiantes & $1 \%$ & $7 \%$ & $32 \%$ & $39 \%$ & $21 \%$ \\
\hline \multicolumn{6}{|c|}{ A partir de los resultados del cuantitativo, julio 2010, elaboración propia. } \\
\hline
\end{tabular}

\begin{tabular}{|c|c|c|c|c|c|}
\hline $\begin{array}{l}\text { Tabla 4. Factor 4. Motivos relacionados con la } \\
\text { afiliación y el interés con las relaciones }\end{array}$ & \multirow[b]{2}{*}{ Nada } & \multirow[b]{2}{*}{ Muy Poco } & \multirow[b]{2}{*}{ Algo } & \multirow[b]{2}{*}{ Bastante } & \multirow[b]{2}{*}{ Mucho } \\
\hline $\begin{array}{l}\text { Creo que como periodista puedo contribuir } \\
\text { a un mundo más justo y mejor / Creo que } \\
\text { como prof. del audiovisual puedo contribuir } \\
\text { a un mundo más justo y mejor / Creo que } \\
\text { como prof. publicidad puedo contribuir a un } \\
\text { consumo más justo. }\end{array}$ & & & & & \\
\hline Periodismo & $1 \%$ & $7 \%$ & $38 \%$ & $27 \%$ & $27 \%$ \\
\hline Comunicación Audiovisual & $2 \%$ & $8 \%$ & $39 \%$ & $34 \%$ & $17 \%$ \\
\hline Publicidad & $1 \%$ & $5 \%$ & $30 \%$ & $32 \%$ & $32 \%$ \\
\hline Total Estudiantes & $1 \%$ & $7 \%$ & $37 \%$ & $32 \%$ & $23 \%$ \\
\hline \multicolumn{6}{|l|}{ Es una profesión nada rutinaria y muy atractiva } \\
\hline Periodismo & $0 \%$ & $0 \%$ & $19 \%$ & $40 \%$ & $41 \%$ \\
\hline Comunicación Audiovisual & $0 \%$ & $2 \%$ & $18 \%$ & $41 \%$ & $39 \%$ \\
\hline Publicidad & $0 \%$ & $5 \%$ & $17 \%$ & $43 \%$ & $35 \%$ \\
\hline Total Estudiantes & $0 \%$ & $2 \%$ & $18 \%$ & $42 \%$ & $38 \%$ \\
\hline \multicolumn{6}{|c|}{ He nacido para ser periodista: no podría dedicarme a otra cosa } \\
\hline Periodismo & $14 \%$ & $20 \%$ & $34 \%$ & $24 \%$ & $8 \%$ \\
\hline Comunicación Audiovisual & $10 \%$ & $14 \%$ & $34 \%$ & $24 \%$ & $18 \%$ \\
\hline Publicidad & $1 \%$ & $21 \%$ & $27 \%$ & $38 \%$ & $13 \%$ \\
\hline Total Estudiantes & $13 \%$ & $19 \%$ & $35 \%$ & $21 \%$ & $12 \%$ \\
\hline \multicolumn{6}{|l|}{ Porque me gusta trabajar en equipo } \\
\hline Periodismo & $1 \%$ & $6 \%$ & $38 \%$ & $39 \%$ & $16 \%$ \\
\hline Comunicación Audiovisual & $2 \%$ & $4 \%$ & $24 \%$ & $45 \%$ & $25 \%$ \\
\hline Publicidad & $2 \%$ & $6 \%$ & $26 \%$ & $41 \%$ & $25 \%$ \\
\hline Total Estudiantes & $2 \%$ & $5 \%$ & $28 \%$ & $42 \%$ & $23 \%$ \\
\hline \multicolumn{6}{|l|}{ Porque siempre he sido una persona creativa } \\
\hline Periodismo & $0 \%$ & $4 \%$ & $28 \%$ & $42 \%$ & $26 \%$ \\
\hline Comunicación Audiovisual & $2 \%$ & $4 \%$ & $20 \%$ & $38 \%$ & $36 \%$ \\
\hline Publicidad & $0 \%$ & $8 \%$ & $21 \%$ & $28 \%$ & $43 \%$ \\
\hline Total Estudiantes & $1 \%$ & $5 \%$ & $22 \%$ & $37 \%$ & $35 \%$ \\
\hline \multicolumn{6}{|c|}{ Me gusta conocer otras culturas y países y esta es una profesión que me lo permitirá } \\
\hline \begin{tabular}{l|l|} 
Periodismo & \\
\end{tabular} & $1 \%$ & $0 \%$ & $12 \%$ & $30 \%$ & $57 \%$ \\
\hline Comunicación Audiovisual & $0 \%$ & $7 \%$ & $26 \%$ & $42 \%$ & $25 \%$ \\
\hline Total Estudiantes & $1 \%$ & $3 \%$ & $19 \%$ & $36 \%$ & $41 \%$ \\
\hline
\end{tabular}

